\title{
Highly efficient coupling in lithium niobate photonic wires by the use of a segmented waveguide coupler
}

\author{
Pierre Aschieri ${ }^{\star}$ and Marc P. de Micheli \\ University of Nice Sophia-Antipolis, LPMC UMR 6622, Parc Valorse, Nice Cedex 2, France \\ *Corresponding author: pierre.aschieri@unice.fr
}

Received 17 February 2011; revised 24 May 2011; accepted 28 May 2011;

posted 31 May 2011 (Doc. ID 142828); published 12 July 2011

\begin{abstract}
The purpose of this article is to show that efficient light coupling in lithium niobate waveguides presenting a strongly confined mode, such as photonic wires, is possible with the use of a periodically segmented waveguide coupler. The coupler consists in an input periodically segmented waveguide whose mode size is adapted to the mode of a standard single-mode fiber coupled to a photonic wire whose mode size is of the same order of the wavelength. The periodic segmentation of the input waveguide allows fulfilling the phase matching condition necessary to achieve an efficient light transfer between these waveguides. The coupling efficiency is typically 5 times higher than the butt-coupled configuration. (C) 2011 Optical Society of America

OCIS codes: $\quad 130.3120,250.3140$.
\end{abstract}

\section{Introduction}

The research effort put on photonic wires is motivated by the fact that strong mode confinement is often looked for in integrated optics to ensure optimum efficiency of the devices. For example, in nonlinear or electro-optic devices, performances are usually proportional to the mode confinement in the waveguide. Unfortunately, in most cases, confinement goes together with high insertion losses. Many solutions have been proposed in the literature to overcome this situation, but their efficiencies are rather poor or are very difficult to implement for technological reasons [1-7]. Tapers based on periodically segmented waveguide (PSW) seem to be an interesting solution, as it does not introduce additional steps in the fabrication process [8-14]. A PSW taper is formed by high-index waveguide segments periodically embedded in a lowindex substrate. The mode size is adjusted by varying the duty cycle of the segmentation defined as the ratio between the length of the high-index segment and the period. Unfortunately, these kinds of tapers are very lossy when the mode is highly confined in the waveguide core, i.e., when the transverse wave-

0003-6935/11/213885-04\$15.00/0

(C) 2011 Optical Society of America guide dimensions are comparable to the wavelength [15], which is the case for a photonic wire. In this paper, we propose use of an electro-optically adjusted segmented directional coupler to efficiently launch the optical power in the highly confined mode of a photonic wire. Despite the fact that the numerical values chosen to demonstrate the interest of the coupler are related to proton exchanged waveguides realized in lithium niobate, the coupler principle is not based on a specific waveguide fabrication process and may be applied to many electro-optic materials.

After a description of the segmented directional coupler principle, the device performances will be calculated for a $\mathrm{LiNbO}_{3}$ proton exchange photonic wire in which the mode size is equal to the wavelength. The calculation indicates that coupling efficiencies between such a photonic wire and a standard telecom fiber as high as $80 \%$ are possible. Limitations will then be discussed and finally, conclusions and perspectives will be drawn.

\section{Device Description}

The device consists in an asymmetric directional coupler as shown in Fig. 1 formed by two waveguides, which have very different modes. The first guide is a photonic wire operating at telecommunication wavelength and obtained using a high-index 


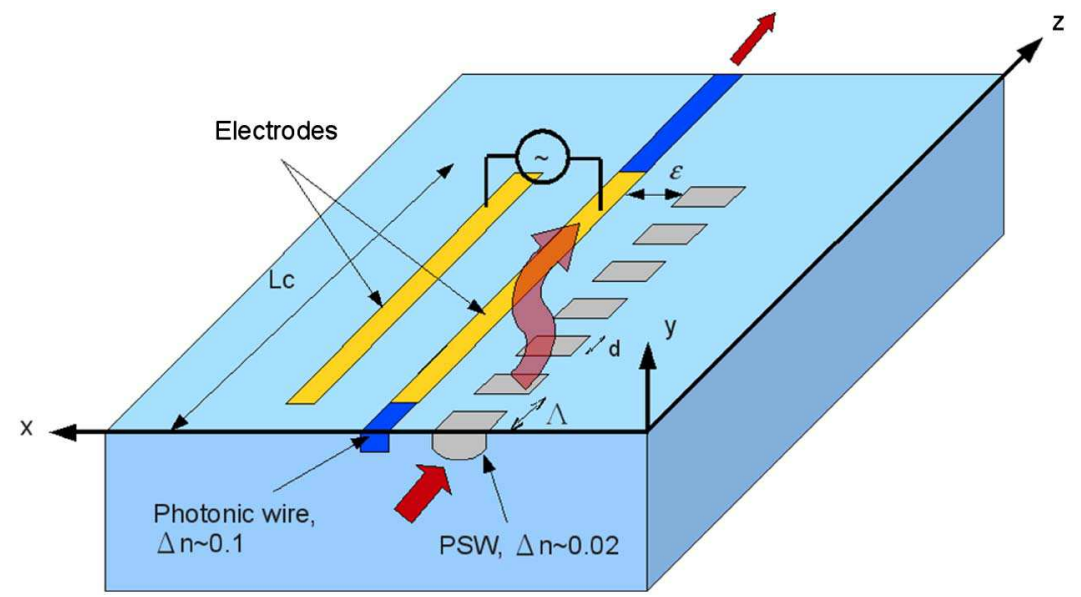

Fig. 1. (Color online) Schematic representation of the segmented coupler. The electro-optic control on the device is done by electrodes represented by stripes on the top of the photonic wire and to the left of it.

difference waveguide $(\Delta n=0.1)$ and small transverse dimensions $(\simeq 1.5 \mu \mathrm{m})$. In this case, more than $80 \%$ of the power is confined in the waveguide core. The second waveguide presents a weakly confined mode showing a good overlap with a standard fiber mode and is achieved using a periodically segmented waveguide where $\Delta n$ is lower, typically between 0.01-0.03. No light is exchanged between these waveguides because of the large difference $\Delta \beta$ between the propagation constants of their modes unless the segmentation period $\Lambda$ of the PSW is chosen in order to compensate for this phase mismatch $\Delta \beta$. The period is then given by the relation

$$
\frac{2 \pi}{\Lambda}=\Delta \beta
$$

or

$$
\Lambda=\frac{\lambda}{n_{h}(\lambda)-n_{\mathrm{psw}}(\lambda)}
$$

where $n_{h}(\lambda)$ is the effective index of the high-index waveguide, $n_{\mathrm{psw}}(\lambda)$ is the effective index of the PSW, and $\lambda$ is the wavelength. If Eq. (2) is satisfied, a complete light transfer between the coupled waveguides can take place. Moreover, the use of a PSW as input waveguide allows optimizing the coupling with the input fiber by adjusting the mode size via a variation of the segmentation duty cycle. This feature is used in PSW tapers and very good performances have been demonstrated experimentally $[10,13,14]$ in the case of soft proton exchange or annealed proton exchange (APE) waveguide fabrication process on $\mathrm{LiNbO}_{3}$ substrate, which gives index increase $\Delta n \simeq 0.02[16,17]$ and where the propagation losses due to the waveguide segmentation are negligible compared to typical waveguide losses.

\section{Device Performances}

In order to estimate the performances of the segmented directional coupler, numerical simulations have been performed using values that are characteristic of $\mathrm{LiNbO}_{3}$ substrate on which it is possible to have a photonic wire and a segmented waveguide. For the photonic wire a proton exchange fabrication process is assumed [18], the waveguide width and depth are both taken equal to $1.4 \mu \mathrm{m}$, the index profile has a step shape and an index difference of $\Delta n=0.12$. Such a waveguide is single mode at $1.5 \mu \mathrm{m}$ and $84.4 \%$ of the power is in the waveguide core. For the PSW, an APE process is supposed, which gives a single-mode waveguide at $1.5 \mu \mathrm{m}$ with a Gaussian index profile both in depth and width direction, with $\Delta n_{\mathrm{psw}}=0.025$ and waveguide width and depth equal to $5 \mu \mathrm{m}$ and $3.5 \mu \mathrm{m}$, respectively. Using a duty cycle $\mathrm{DC}=0.5$ gives an overlap of $90 \%$ with a $8 \mu \mathrm{m}$ diameter Gaussian mode typical for a standard telecom fiber. The effective indices are $n_{h}=2.19529$ and $n_{\mathrm{PSW}}=2.14188$ for the high-index waveguide and the PSW, respectively. In order to have a total light transfer between the two waveguides, according to Eq. (2), the required segmentation period is $\Lambda=2 \overline{8} .08 \mu \mathrm{m}$. The gap between waveguides is fixed at $\varepsilon=3.2 \mu \mathrm{m}$; justification of this value will be provided in the next section concerning propagation losses. The whole device has been simulated with a 3D FD-BPM code and Fig. 2 represents the amplitude mode distribution transfer from the input PSW waveguide $[Z=0$, Fig. 2(a)] to the high-index output waveguide during propagation. After $Z=2 \mathrm{~mm}$ [Fig. 2(b)] of propagation, the light is partially coupled into the high-index waveguide and when the propagation distance is $Z=8 \mathrm{~mm}$ [Fig. 2(c)], which correspond to the coupling length $L c$, all the light is transferred into the high-index output waveguide. Ninety percent of the power is transferred from one waveguide to the other. The $10 \%$ difference is due to the propagation losses in the segmented waveguide. Therefore, despite the predicted losses, using the asymmetric coupler allows improving the coupling by at least a factor 5 .

\section{A. Propagation Losses}

Some further comments have to be made on the predicted propagation losses. A typical value for the propagation losses of a PSW taken alone is 

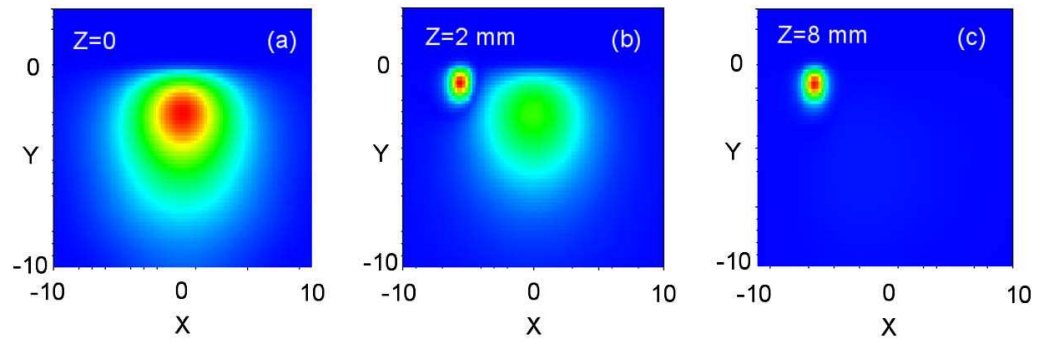

Fig. 2. (Color online) Evolution of the light transfer at the input waveguide $(Z=0)$, after $Z=2 \mathrm{~mm}$ of propagation and at the output photonic wire $(Z=8 \mathrm{~mm})$, transverse dimensions $X$ and $Y$ are in $\mu \mathrm{m}$.

$0.1 \mathrm{~dB} / \mathrm{cm}$ but the presence of the photonic wire leads to extra propagation losses, whose value becomes $0.5 \mathrm{~dB} / \mathrm{cm}$. This is explained by the fact that the low-loss eigenmode of the PSW is perturbed by the photonic wire, which induces coupling into the radiation modes. Figure 3 represent the propagation losses of the segmented coupler as a function of the gap $\varepsilon$ between waveguides. As expected, an exponential decrease of the losses occurs as the spacing increase, but this induces an exponential increase of the coupling length. The value of $\varepsilon=3.2 \mu \mathrm{m}$ used for the gap between the waveguides in the previous section corresponds to a good compromise. In order to further reduce the intrinsic propagation losses of the PSW, one can increase the transverse dimensions of the waveguide [15], but this is possible only in the range where the waveguide remains single mode. The segmentation period $\Lambda$ is also a critical parameter for the propagation losses, and, according to previous work $[9,13]$, the segmentation period should not be too large. Since the segmentation period is fixed by the phase matching condition given by Eq. (2), a wavelength reduction or an increase of the effective index difference leads to a reduction of the segmentation period $\Lambda$, which might be a better situation regarding the losses. In other words, the more the mode is confined in the photonic wire, the lower are the losses and better is the power transfer between waveguides. Nevertheless, a lower limit for the period is fixed by fabrication constraints and the

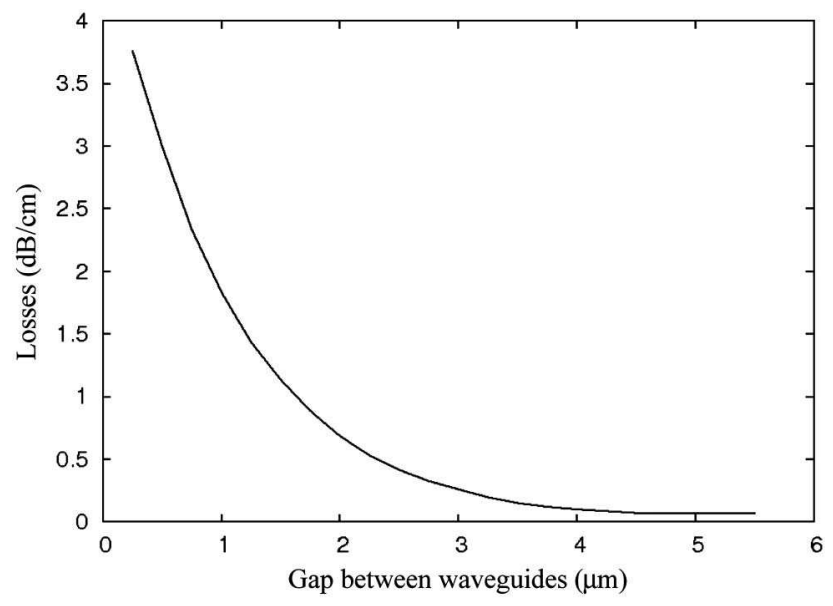

Fig. 3. Losses as a function of the gap $\varepsilon$ between waveguides. guiding segments of the PSW cannot be smaller than typically $1 \mu \mathrm{m}$.

\section{B. Tolerances to Parameters Variations}

Directional couplers are very sensitive devices. In the case of segmented directional couplers, in which a phase matching condition is involved, they are even more sensitive to the different parameters. Simulations have then been performed in order to estimate the normalized power transfer from the input to the output of the device. Figure 4 represents the power transfer as a function of the wavelength and it is clear that the device is very sensitive to wavelength. For a coupling length of $4 \mathrm{~mm}$ the full width at halfmaximum (FWHM) is $4 \mathrm{~nm}$, and it is reduced to $2 \mathrm{~nm}$ if the coupling length is increased to $L c=8 \mathrm{~mm}$. This wavelength dependence is a classical feature of devices based on a grating, such as Bragg reflector or long period fiber grating, for example, and it is well known that wavelength FWHM is proportional to $1 / L c[19,20]$. One should note that the maximum power transfer, which is limited by the losses, does not vary with the coupling length. Indeed, when the distance between the waveguides is reduced, the loss rate and the coupling rate increase and the coupling length is reduced. The total losses of a structure designed to perform a maximum power transfer are, therefore, more or less a constant. In Fig. $\underline{5}$, we plot the variation of the power transfer as a function of an

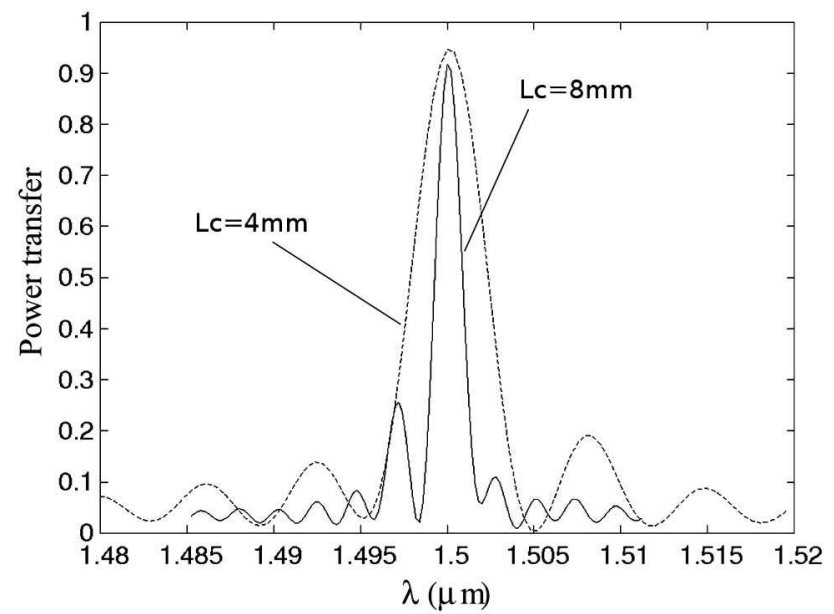

Fig. 4. Power transfer as a function of the wavelength variation for two different coupling lengths $L_{c}$. 


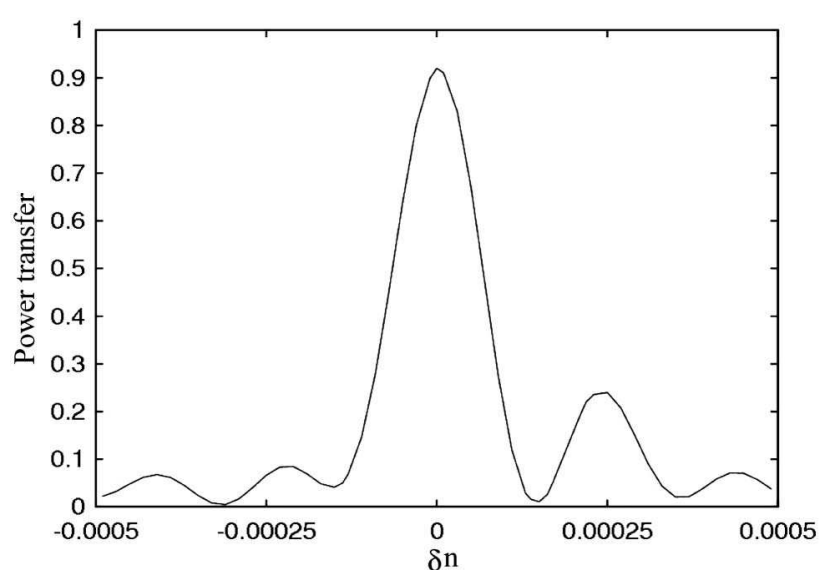

Fig. 5. Power transfer as a function of the index variation $\delta n$ of the photonic wire.

index variation $\delta n$ of the high-index waveguide, such as $n_{h} \rightarrow n_{h}+\delta n$. The power transfer is very sensitive to this index variation $\delta n$, which induces a deviation from the phase matching condition $\Delta \beta=0$. The FWHM is only $\delta n=10^{-4}$, which is smaller than the accuracy of the index variation obtained experimentally. Therefore, for a practical device, it will be necessary to add electrodes to fine-tune the waveguide optical index in order to perfectly satisfy the phase matching condition. The required index variation is very weak and a low voltage control will be sufficient to compensate the phase mismatch.

\section{Conclusion}

It this paper, we present the principle of a segmented directional coupler and its performances when it is used to achieve an efficient power injection into a photonic wire. The light is coupled into an input PSW and then transferred to the photonic wire through the periodic index segmentation of the PSW to fulfill the phase matching condition. The transfer efficiency can be as high as $90 \%$ for typical waveguides fabricated on $\mathrm{LiNbO}_{3}$ as the use of a PSW also allows adjusting the input mode size to achieve a good overlap (90\%) with the fiber mode. Therefore, a power coupling from the input fiber to the photonic wire of $80 \%$ can be expected. The phase matching condition required to obtain the maximum power transfer is very sensitive and in practice it will be necessary to add electrodes along the waveguides to adjust the phase mismatch. One should also note that the phase matching sensitivity of this segmented directional coupler can be used to realize a wavelength filter or a sensor, but more numerical work will have to be performed to that aim.

This work has been carried out with the financial support of the ANR project phoXcry nANR-09NANO-004 funded by the French National Agency (ANR) in the framework of its 2009 program in Nanosciences, Nanotechnologies and Nanosystems (P3N2009).

\section{References}

1. J. Albert and G. L. Yip, "Insertion loss reduction between single-mode fibers and diffused channel waveguides," Appl. Opt. 27, 4837-4843 (1988).

2. M. Itoh, T. Saida, Y. Hida, M. Ishii, Y. Inoue, Y. Hibino, and A. Sugita, "Large reduction of singlemode-fibre coupling loss in $1.5 \% \Delta$ planar lightwave circuits using spot-size converters," Electron. Lett. 38, 72-74 (2002).

3. B. L. Sopori, C. M. Phillips, and W. S. C. Chang, "Efficient optical waveguide coupler," Appl. Opt. 19, 790-801 (1980).

4. P. G. Suchoski and R. V. Ramaswamy, "Constant-width variable-index transition for efficient $\mathrm{Ti}: \mathrm{LiNbO}_{3}$ waveguide-fiber coupling," J. Lightwave Technol. 5, 1246-1251 (1987).

5. K. Kasaya, O. Mitomi, M. Naganuma, Y. Kondo, and Y. Noguchi, "A simple laterally tapered waveguide for low-loss coupling to single-mode fibers," IEEE Photon. Technol. Lett. 5, 345-347 (1993).

6. B. Luyssaert, P. Vandersteegen, and R. Baets, "Efficient nonadiabatic planar waveguide tapers," J. Lightwave Technol. 23, 2462-2468 (2005).

7. F. Van Laere, D. Taillaert, D. Van Thourhout, and R. Baets, "Compact and highly efficient grating couplers between optical fiber and nanophotonic waveguides in bonded InP-membranes," in 32nd European Conference on Optical Communication (IEEE, 2006), paper Tu1.4.5.

8. Z. Weissman and A. Hardy, "2-D mode tapering via tapered channel waveguide segmentation," Electron. Lett. 28, 1514-516 (1992).

9. Z. Weissman and I. Hendel, "Analysis of periodically segmented waveguide mode expanders," J. Lightwave Technol. 13 2053-2058 (1995).

10. M. H. Chou, M. A. Arbore, and M. M. Fejer, "Adiabatically tapered periodic segmentation of channel waveguides for modesize transformation and fundamental mode excitation," Opt. Lett. 21, 794-796 (1996).

11. F. Dorgeuille, B. Mersali, S. Francois, G. Herve-Gruyer and M. Filoche, "Spot size transformer with a periodically segmented waveguide based on InP," Opt. Lett. 20, 581-583 (1995).

12. M. M. Spuhler, B. J. Offrein, G. Bona, R. Germann, I. Massarek, and D. Ern, "A very short planar silica spot-size converter using a nonperiodic segmented waveguide," J. Lightwave Technol. 16, 1680-1685 (1998).

13. D. Castaldini, P. Bassi, S. Tascu, P. Aschieri, M. P. De Micheli, and P. Baldi, "Soft-proton-exchange tapers for low-insertion loss $\mathrm{LiNbO}_{3}$ devices," J. Lightwave Technol. 25, 1588-1593 (2007).

14. D. Castaldini, P. Bassi, P. Aschieri, S. Tascu, M. De Micheli, and P. A. Baldi, "High performance mode adapters based on segmented SPE:LiNbO 3 waveguides," Opt. Express 17, 17868-17873 (2009).

15. Z. Weissman and A. Hardy, "Modes of periodically segmented waveguides," J. Lightwave Technol. 11, 1831-1838 (1993).

16. L. Chanvillard, P. Aschieri, P. Baldi, D. B. Ostrowsky, M. De Micheli, L. Huang, and D. J. Bamford, "Soft proton exchange on PPLN: a simple waveguide fabrication process for highly efficient non-linear interactions," Appl. Phys. Lett. 76, 1089-1091 (2000).

17. M. L. Bortz and M. M. Fejer, "Annealed proton-exchange $\mathrm{LiNbO}_{3}$ waveguides," Opt. Lett. 16, 1844-1846 (1991).

18. M. De Micheli, J. Botineau, S. Neveu, P. Sibillot, and D. B. Ostrowsky, "Independent control of index and profile in protonexchanged lithium niobate guide," Opt. Lett. 8, 114-115 (1983).

19. T. Erdogan, "Cladding-mode resonances in short- and long- period fiber grating filters," J. Opt. Soc. Am. 14, 1760-1773 (1997).

20. B. S. Kawasaki, K. O. Hill, D. C. Johnson, and Y. Fujii, "Narrowband Bragg reflectors in optical fibers," Opt. Lett. 3, 66-68 (1978). 